\title{
A spaceborne synthetic aperture radiometer simulated by the TUD demonstration model
}

\author{
Laursen, Brian; Skou, Niels
}

Published in:

Proceedings of the International Geoscience and Remote Sensing Symposium

Link to article, DOI:

10.1109/IGARSS.1994.399426

Publication date:

1994

Document Version

Publisher's PDF, also known as Version of record

Link back to DTU Orbit

Citation (APA):

Laursen, B., \& Skou, N. (1994). A spaceborne synthetic aperture radiometer simulated by the TUD

demonstration model. In Proceedings of the International Geoscience and Remote Sensing Symposium (Vol. Volume 3, pp. 1314-1316). IEEE. https://doi.org/10.1109/IGARSS.1994.399426

\section{General rights}

Copyright and moral rights for the publications made accessible in the public portal are retained by the authors and/or other copyright owners and it is a condition of accessing publications that users recognise and abide by the legal requirements associated with these rights.

- Users may download and print one copy of any publication from the public portal for the purpose of private study or research.

- You may not further distribute the material or use it for any profit-making activity or commercial gain

- You may freely distribute the URL identifying the publication in the public portal 


\title{
A Spaceborne Synthetic Aperture Radiometer Simulated by the TUD Demonstration Model
}

\author{
Brian Laursen \& Niels Skou
}

Electromagnetics Institute, B 348, Technical University of Denmark (TUD) DK-2800 Lyngby, Denmark. Phone: (45) 428814 44, Fax: (45) 45931634

\section{ABSTRACT}

The TUD Synthetic Aperture Radiometer demonstration model consists of a 2-channel X-band correlation radiometer with two horn antennas and an antenna mounting structure enabling the horns to be mounted in relevant positions within a certain aperture. The cross correlation of the signals from the 2 antennas produces a sample of the visibility function, which is the Fourier transform of the brightness temperature distribution of the target area. A total aperture synthesis is obtained by sequentially placing the two antenna elements in all required pairs of positions and measure the corresponding samples of the visibility function followed by an image reconstruction procedure which is based on the inverse Fourier transform.

The system has been used to simulate a spaceborne instrument (MIRAS - see other papers in this conference) in order to validate the image reconstruction processing.

\section{INTRODUCTION}

The Synthetic Aperture Radiometer (SARad) systems are fundamentally different from traditional radiometer systems in that they measure in the spatial frequency domain and an inverse Fourier transformation is needed to obtain the normal brightness temperature distribution in the spatial domain. Aperture synthesis involves interferometric measurements made by cross-correlating the signals from two spatially separated antennas having overlapping field-of-view.

The cross-correlation of the signals from the two antennas (having passed through two properly designed radiometers) produces a sample of the visibility function corresponding to the relative positions of the antenna elements. By making a new measurement with another relative position of the antennas, another sample of the visibility function is found. Only a limited number of measurements i. e. positions are required to adequately sample the visibility function. In a practical imaging system this is done by having a proper number of (small) antenna elements placed properly within the aperture and then simultaneously crosscorrelate all possible pairs to generate the visibility function.

The TUD (Technical University of Denmark) demonstration model consists of a 2-channel X-band correlation radiometer with two horn antennas and an antenna mounting structure enabling the horns to be mounted in relevant positions within a certain aperture. A total aperture synthesis is obtained by sequentially placing the two antenna elements in all required pairs of positions and measure the corresponding samples of the visibility function. The demonstration model thus requires the test scene to remain stable for a considerable time - typically a few hours.

The purpose of such a 2-channel demonstration model is twofold: with relatively cheap instrumentation the synthetic aperture radiometer concept can be demonstrated and investigated, and specific configurations for air- or spaceborne systems can be simulated and evaluated while they are still in the design phase.

\section{ABOUT SARad THEORY}

The theory concerning synthetic aperture radiometry, is published in (Thompson et al, 1986) and (Ruf et al, 1988) and shall not be discussed here; only a few items are commented upon. In general it is assumed that the object to be sensed is far away from the antenna (in the far-field of the synthetic aperture (DSYN) i.e. $r>20 \mathrm{D}_{\mathrm{SYN}^{2}} / \lambda$ ). However, it can be shown that the general theory also covers the case where the object is in the near field provided that the antenna is focused by mounting the antenna elements on a section of a sphere with the object at the centre and provided that the elements are placed symmetrically about the centre of the aperture (Laursen et al, 1994).

\section{THE TUD DEMONSTRATION MODEL}

\section{The Block Diagram}

The radiometer system employs two single-sideband superheterodyne receivers, see Figure 1. The RF centre frequency is $10.3 \mathrm{GHz}$ and the IF centre frequency is $300 \mathrm{MHz}$ with a 150 $\mathrm{MHz}$ system bandwidth. The noise figure is $4.7 \mathrm{~dB}$. Horn antennas are used as antenna elements and phase stable flexible microwave cables are used between the antennas and the receivers. A common local oscillator ensures channel coherence. The phase shifter between the LO and the mixer in channel 2 is used to compensate for any difference in electrical delay in the two receivers.

The power divider network used to feed the analogue correlators consists of quadrature hybrids followed by in-phase 2 way power dividers. The analogue correlator is a four quadrant analogue multiplier followed by an integrator. Finally the correlation radiometer also includes a detection channel used for measuring the spatial DC frequency and used for calibration.

The electronics require calibration of the gain and noise figure every $10-15$ minutes, and two reference temperatures are used. The sky is used as a cold reference temperature by pointing the antenna element at zenith. As a warm reference temperature the antenna element is pointed at absorbers.

In order to achieve identical electrical delays in the two channels the phase of the local oscillator signal in channel 2 is adjusted to maximize the I output signal (minimize the $Q$ output) for equal, correlated input signals. These signals are generated by a common noise source $T_{2}$ and a Wilkinson power divider. $T_{2}$ must be different from the physical temperature of the power divider to obtain two correlated output signals of the power divider. The reason for this is the noise signal from the internal

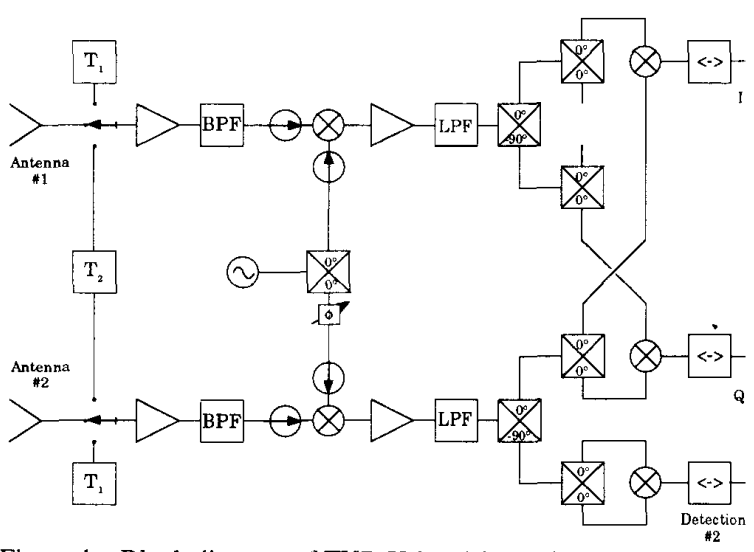

Figure 1. Block diagram of TUD X-band SARad 
resistor in the power divider being $180^{\circ}$ out of phase on the output ports. When $T_{2}$ equals the temperature of the power divider, the effect of the two noise signals cancels in correlation.

The reference temperatures $T_{1}$ are used as uncorrelated input signals in the two channels.

\section{The Near-Field Antenna}

In this configuration the demonstration model is nadir looking. The experiments are carried out outdoors, and the scene consists of metal targets reflecting the sky (low brightness temperature) against a warm, uniform background. In the example to be discussed later metal letters spelling "EMI" have been used as targets.

The antenna is focused by having the antenna elements, which are rectangular horns, mounted on a curved rail, see Figure 2 . The horns are always mounted symmetrically about the centre of the rail.

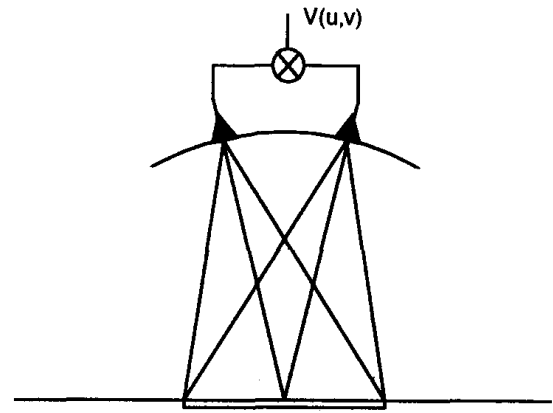

Figure 2. Focused antennas in a near field synthetic aperture.

By rotating this rail around a vertical axis, the full aperture can be covered. The circular shaped rail ensures that all spatial frequencies are exactly centred, and this is necessary when an inverse Fourier transform is used in the image reconstruction on data from a near field measurement.

The visibility function is sampled with a $2 \lambda$ spacing on the rail. When the rail is rotated, the angular sampling distance corresponds to a $2 \lambda$ spacing between visibility samples on the largest circle in the $(u, v)$ plane, see Figure 3 . The $(u, v)$ coordinates are the distances between the antenna elements measured in wavelengths.

The lowest-frequency visibility sample cannot be measured because of the size of the antenna element. The samples on the ring nearest to the centre are therefore set to zero.

\section{The Far-Field Antenna}

Even a modest aperture size require the target to be more than $100 \mathrm{~m}$ away so nadir looking become difficult. Also, more complex artificial targets become unrealistic and one have to resort to imaging the horizon with some distinctive buildings as target. In the example to be discussed later a power plant with a tall concrete chimney has been imaged. The antenna aperture is now plane and in the present case the horns have been mounted along the arms of a Y-shaped structure. A $1 \lambda$ spacing between possible positions on the arms was used. The coverage in the $(u, v)$ plane is shown in Figure 4. Again, the lowest-frequency visibility samples cannot be measured due to the size of the antenna elements.

\section{IMAGE RECONSTRUCTION}

\section{Correction of Common Correlated Noise}

Each visibility sample needs a correction of the offset error corresponding to the output of the correlator when the input signals to the two channels are completely uncorrelated. This error signal is probably caused by the fact that the two radiometer channels are using the same local oscillator and the same power supply hence some common - correlated - noise is always present.

Interpolation

The visibility samples must be interpolated from the measured $(u, v)$ grid to a rectangular grid suitable for an inverse Fourier transform.

In the near-field case the visibility samples at the first square of the rectangular $(\mathrm{u}, \mathrm{v})$ grid are set to zero because of the missing frequencies. Visibility samples on the inner rings are oversampled because of the constant angular samplings distance. On the basis of the missing frequencies at the first ring and the oversampling on the following rings, a nearest neighbour interpolation routine is used to interpolate visibility samples on the second square of the rectangular $(u, v)$ grid. For all other rectangular $(u, v)$ samples within a radius corresponding to synthesised aperture, a third order Lagrange interpolation routine is used.

In the far-field case a one dimensional interpolation of the visibility samples is required. All the rectangular $(u, v)$ samples with an odd $u$-value is determined by interpolation along a line parallel to the v-axis, see Figure 4. A sixth order Lagrande interpolation routine is used.

Also in the far-field case, the lowest visibility samples are not measured because of the size of the antenna elements. An extrapolation of these missing spatial frequencies from the measured spatial frequencies is performed. This is possible for the image of the chimney since the structure in the brightness temperature variation is simple; in the vertical plane the brightness temperature variation is mainly given by the horizon, and in the horizontal plane it is mainly given by the chimney and partly by the relatively small power plant. This relatively simple brightness temperature image is giving a visibility function with a slow variation as function of the spatial frequency co-ordinates.

\section{Inverse transform}

After multiplication with an appropriate window the visibility function is inverse Fourier transformed to obtain the brightness distribution of the scene. The choice of window depends on the requirements to the spatial resolution and the beam efficiency of the synthesised beam; the EMI image is processed with a Hann window $(\alpha=1.2)$ and the chimney image is processed with a Hamming window.

Finally an antenna element gain correction, followed by a ground projection, is performed to obtain a true image of the measured brightness temperature distribution as a function of ground co-ordinates.

\section{DISCUSSION OF THE IMAGERY}

In Figure 5 it is seen how the metal letters spelling "EMI" are successfully imaged by the near-field set-up. The "altitude" was $1.4 \mathrm{~m}$, the aperture $22 \lambda(66 \mathrm{~cm})$, the synthetic beamwidth $3^{\circ}$, the field-of-view $30^{\circ}$ resulting in 10 by 10 pixels.

It is seen that the temperature rise to more than $400 \mathrm{~K}$ at the edge of the image and especially in the corners. This is an aliasing effect in the spatial domain (the brightness temperature domain) caused by undersampling in the spatial frequency domain (the visibility function domain). The required sampling distance in the spatial frequency domain is determined by the antenna element radiation pattern. It is well known that a small aliasing only influence the edge of the image, and not the centre, as it is seen on the images.

Also the power plant with the chimney is relatively successfully imaged, in this case by the far-field set-up, see Figure 6 . The synthetic aperture $(20 \lambda$ or $60 \mathrm{~cm})$ is placed at a distance of 150 meters from the chimney leading to a nine meters resolution. The spatial frequency sampling is $1 \lambda$, but the field-of-view is limited by the radiation pattern of the antenna elements, and is chosen to $\pm 25^{\circ}$ (where the gain is down by $8 \mathrm{~dB}$ ). This is giving a image size of 15 by 15 pixels.

The chimney is a point target in the $\mathrm{x}$-axis direction since the width is only $3.5 \mathrm{~m}$, and the temperature of the concrete chimney 
is measured to be $170 \mathrm{~K}$ with a $-3 \mathrm{~dB}$ width of ten meters. Taking into consideration the width of the chimney compared to the resolution, the measured temperature of the chimney is what would be expected.

\section{CONCLUSIONS}

Using rather simple hardware, it has been possible to demonstrate the 2-dimensional synthetic aperture radiometer concept and to experience some of the problem areas. A nex generation system is presently under development. Great emphasis will be put to long term stability recalling the duration of a typical measurement. Calibration issues will be the prime objective for the new system.

\section{ACKNOWLEDGEMENT}

The work has been supported by the European Space Agency and the Danish National Science Foundation.

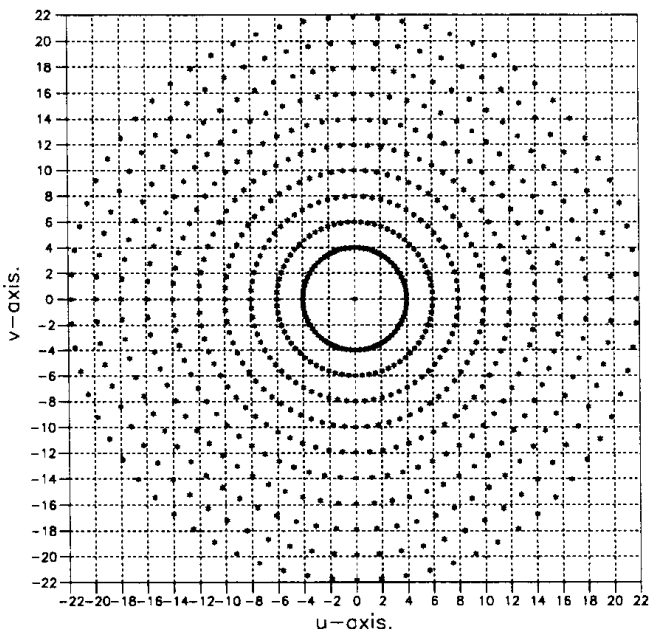

Figure 3. Measured spatial $(u, v)$ frequencies for the near-field set-up and a rectangular grid (the grid line intersections correspond to the sampling points needed for an inverse Fourier transform.

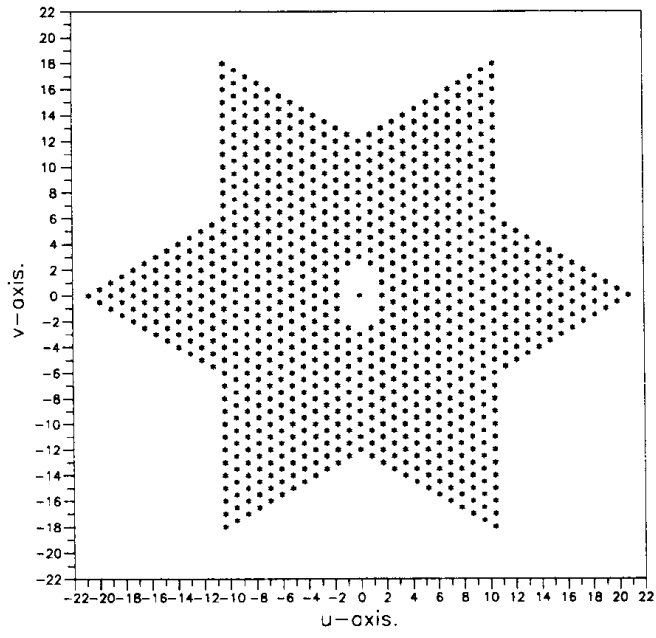

Figure 4. Measured spatial $(u, v)$ frequencies for the far-field setup, with $1 \lambda$ sampling distance (grid not shown).

\section{REFERENCES}

Davidheiser, R. A., TRW: personal communication.

Laursen, B., Pedersen, H.M., Skou, N.: "The TUD Synthetic Aperture Radiometer Demonstration Model," in Proc. of the Specialist Meeting on Microwave Radiometry and Remote Sens. of the Environment, Rome, Feb. 1994.

Ruf, C.S., Swift, C.T., Tanner, A.B., Le Vine, D.M.: "Interferometric Synthetic Aperture Microwave Radiometry for the Remote Sensing of the Earth." IEEE Trans. Geoscience and Remote Sens., vol.26 no.5, p.597-611, Sept. 1988

Thompson, A.R., Moran, J.M., Swenson, G.W.: "Interferometry and Synthesis in Radio Astronomy." JOHN WILEY \& SONS, 1986

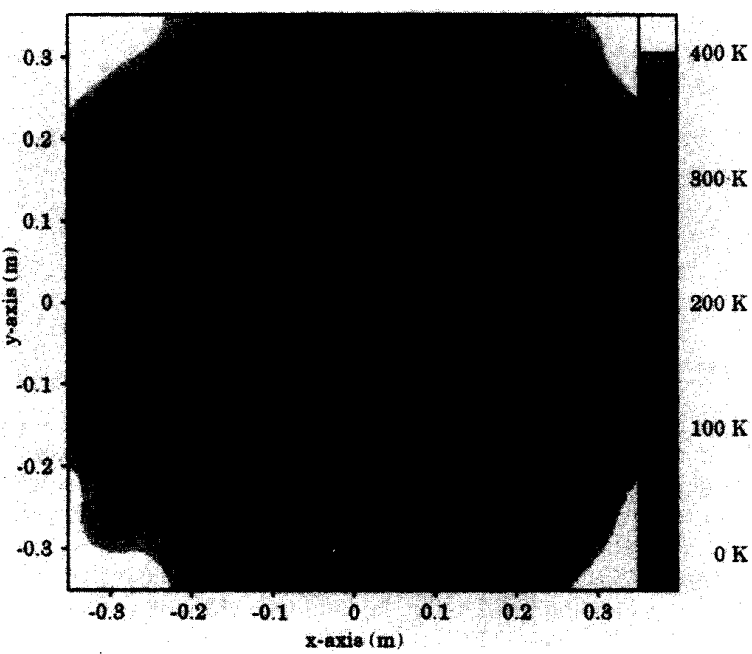

Figure 5. Metal letters spelling "EMI" are imaged by the nearfield set-up.

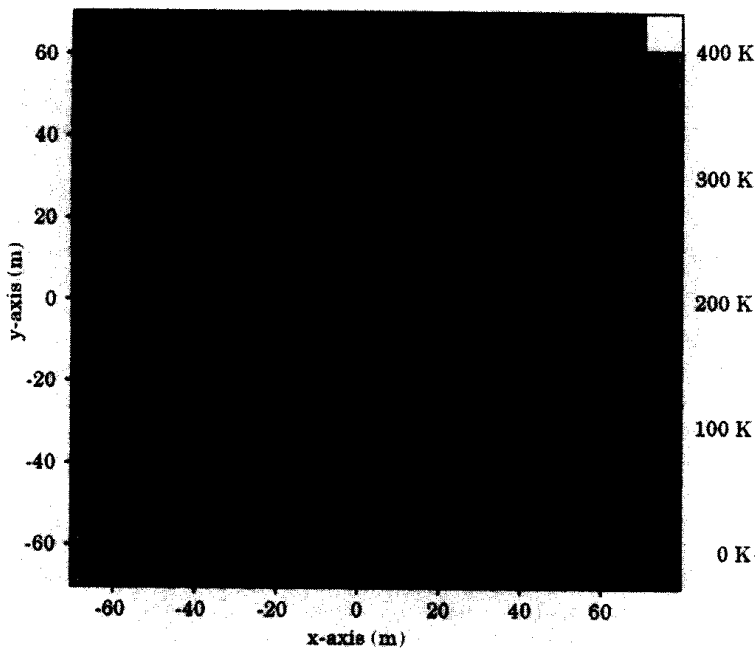

Figure 6. A power plant with a chimney is imaged by the farfield set-up. 